\title{
Non-Linear Analysis of Bubbling Fluidized Beds
}

\author{
A. Vaidheeswaran ${ }^{1, a)}$ and S. Rowan ${ }^{1, b)}$ \\ National Energy Technology Laboratory, 3610 Collins Ferry Rd, Morgantown, \\ WV 2650\%, USA
}

(Dated: 26 June 2020)

Results from non-linear analysis of bubbling fluidized beds are presented in this study. The experiments were performed in cylindrical columns having internal diameters of 2.5 inches, 4 inches and 6 inches while operating conditions, material properties and static bed height were held constant. Superficial velocity of air at the inlet was varied from 2.97 to 5.35 times minimum fluidization velocity in each column. The test procedure involved randomization and replication to estimate measurement uncertainty and identify bias if present. The columns were split into regions based on dominant physical mechanisms occurring within. Fractal parameters were evaluated from differential pressure data which confirm deterministic chaos. These measures represent a broad range of spatial and temporal scales and were used to elucidate multiphase dynamics in different sections of these columns. Fractal analysis is hence shown to provide more intuition particularly when a true scale-up study based on non-dimensional groups becomes prohibitive.

\footnotetext{
a) Also at Leidos Research Support Team, 3610 Collins Ferry Rd, Morgantown, WV 26507, USA; Electronic mail: avinash.vaidheeswaran@netl.doe.gov

b) Also at Leidos Research Support Team, 3610 Collins Ferry Rd, Morgantown, WV 26507, USA
} 


\section{INTRODUCTION}

Fluidization is caused by dynamic equilibrium between gravity and drag, which is known to enhance mixing and heat transfer characteristics. Gas flowing through a granular medium results in non-linear gas-particle and particle-particle interactions leading to a range of spatio-temporal scales which are not linearly superimposable. At the micro-scale, particles collide with other particles and walls of a column which dissipate energy due to their inelastic nature. On the other hand, spatial inhomogeneities exist in the form of bubbles at the meso-scale where particle concentration approaches zero. These propagate as concentration waves while enhancing stream-wise and lateral transport of particles as well as providing paths of least resistance to the gas-phase. Clusters and streamers are other meso-scale structures which are formed due to a combination of hydrodynamic instability and inelastic inter-particle collisions at higher flow rates. Characterizing these mechanisms is important in determining the overall performance of a fluidized bed reactor. It is however challenging to analyze a system having such a high degree of non-linearity using a naive approach. Understanding multi-phase hydrodynamics is also essential for successful scale-up of systems from laboratory-scale to pilot-scale and industrial-scale. Though scaling laws have been proposed for fluidized bed systems ${ }^{1}$, feasibility remains an issue while using non-dimensional groups derived from continuum-based governing equations. This may require changing properties of particulate- and carrier-phase along with operating conditions among other control parameters. Hence, geometric scale-up study in gas-solid flows without considering hydrodynamic scaling laws is more common in literature, which is followed in the work presented.

Non-linear analysis often relies on transformation from physical space to spectral- or phase-space. Development of mechanistic tools for chaos has made it possible to provide more granularity in complex multi-phase systems in engineering applications ${ }^{2-6}$ and nature $^{7}$. Previous use of this technique in gas-solid fluidization includes works of Pence and Beasley $^{8}$, Zhao and Yang ${ }^{9}$, Blomgren et al. ${ }^{10}$, Fullmer and Hrenya ${ }^{11}$, Higham, Shahnam,

and Vaidheeswaran ${ }^{12}$. Obtaining high-quality experimental data with measurement uncertainty has also been a major challenge in granular and multi-phase flows Gel et al. ${ }^{13}$. A systematic approach has been adopted recently by Gopalan et al. ${ }^{14}$ and Vaidheeswaran, Shaffer, and Gopalan ${ }^{15}$ which involve replicates and randomization in the design of experiments. This is critical in identifying individual contributions of uncertainties from different 
sources including instrumentation and chaos in the system. Therefore, a similar testing procedure is followed in the current study.

In this work, non-linear characteristics in three different bubbling fluidized bed columns are described using time series of differential pressure signals. As the name suggests, the most dominant meso-scale structures in such units are bubbles which represent regions of void or negligible particle concentration. Pressure signals inside a bubbling fluidized bed originate due to multiple reasons ${ }^{16}$ including: (i) formation, coalescence and breaking of bubbles, (ii) bubble eruption at the interface, (iii) passage of bubbles and (iv) interactions between fluidized particles. These are prone to being amplified or attenuated depending on the properties of emulsion phase and locations of pressure ports. As opposed to absolute pressure, differential pressure has the ability to filter out fluctuations outside the region of interest and represent the dynamics within ${ }^{16}$. Data were recorded across distributor plate, dense region, dilute region and combination of dense and dilute regions. Measures from chaos and recurrence quantification analysis (RQA) are used for a qualitative description of features in these columns, although not hydrodynamically scaled. The remainder of the article is organized as follows: Details regarding experimental set-up and procedure are

outlined in Section II. Discussion on estimated fractal measures is provided in III followed by conclusions drawn from this study.

\section{SETUP AND PROCEDURE}

Experiments on bubbling fluidization were performed using the cylindrical columns depicted in Figure 1. The initial static bed height was set to $15.24 \mathrm{~cm}$ in each case which translates to $0.79 \mathrm{~kg}, 1.90 \mathrm{~kg}$ and $3.74 \mathrm{~kg}$ for the 2.5-inch, 4-inch and 6-inch units respectively. Experiments were performed using glass particles having a density of $2510 \mathrm{~kg} / \mathrm{m}^{3}$ and Sauter mean diameter of $332 \mu \mathrm{m}$ which are classified under Group B based on Geldart's criterion ${ }^{17}$. Dry air at atmospheric conditions was used for fluidizing the particles. A characteristic velocity in such systems is the minimum fluidization velocity, $U_{m f}$ which represents transition from a packed to fluidized state. Rao et al. ${ }^{18}$ determined the effect of channel diameter on $U_{m f}$, and observed a drop in its value with reduction in wall friction as diameter increases. $U_{m f}$ values obtained in this study are summarized in Table I. 
TABLE I. Minimum fluidization values from test sections used in this study

\begin{tabular}{cc}
\hline \hline Internal diameter of column (inches) & $U_{m f}(\mathrm{~m} / \mathrm{s})$ \\
\hline 2.5 & 0.079 \\
4.0 & 0.073 \\
6.0 & 0.070 \\
\hline \hline
\end{tabular}

TABLE II. Instrumentation range (in $\mathrm{Pa}$ ) used in different test sections

\begin{tabular}{lccr}
\hline \hline Measurement & 2.5 -inch & 4 -inch & 6-inch \\
\hline$\Delta P_{1}=P_{0}-P_{1}$ & 3732.60 & 3732.60 & 7465.19 \\
$\Delta P_{2}=P_{1}-P_{2}$ & 2488.40 & 2488.40 & 2488.40 \\
$\Delta P_{3}=P_{2}-P_{3}$ & 3732.60 & 3732.60 & 3732.60 \\
$\Delta P_{4}=P_{3}-P_{4}$ & 1244.20 & 1244.20 & 3483.76 \\
$\Delta P_{5}=P_{4}-P_{5}{ }^{\text {a }}$ & - & 622.10 & 622.10 \\
$\Delta P_{5}=P_{1}-P_{5}{ }^{\mathrm{b}}$ & 3732.60 & - & - \\
$\Delta P_{6}=P_{1}-P_{5}{ }^{\mathrm{c}}$ & - & 3732.60 & 3732.60 \\
\hline \hline
\end{tabular}

\footnotetext{
a 4-inch \& 6-inch

b 2.5-inch

c 4 -inch \& 6-inch
}

Further details regarding minimum fluidization experiments have been avoided for the sake of brevity. Henceforth, $U_{m f}$ refers to the value corresponding to the 2.5-inch unit unless otherwise stated.

Pressure transducers are used to record differential pressure along different sections in the cylindrical columns. Table II summarizes information regarding instrumentation in different units. Air at the inlet is introduced through a mass flow controller at five superficial velocities viz., $[2.97,3.57,4.16,4.76,5.35] U_{m f}$. During the experiments, these settings were randomized and five replicates were introduced for each case to quantify uncertainty in measurements. Pressure values were recorded for each test for a duration of $180 \mathrm{~s}$. Initial transients were removed during analysis to ensure stationarity in a statistical sense.

To facilitate better understanding, the respective test sections are divided into four regions viz., distributor, dense, dilute and combined based on the location of pressure ports. 
The names have been chosen for the purpose of distinguishing while relaxing their strict definitions. Distributor corresponds to the region between pressure ports on either side of the distributor plate. Dense region corresponds to the dense portion where particle concentration is relatively higher. Dilute region refers to the section covering interface between the dense bed and freeboard where bubbles erupt. Combined region is a combination of dense and dilute regions as defined. In terms of measurements, differential pressure across distributor corresponds to $\Delta P_{1}$. Pressure drop across dense region corresponds to $\Delta P_{2}+\Delta P_{3}$ for the 2.5-inch column, and $\Delta P_{2}$ for the 4-inch and 6-inch columns. For the 2.5-inch column, differential pressure across dilute region corresponds to $\Delta P_{4}$ and combined region corresponds to $\Delta P_{5}$, while they correspond to $\Delta P_{3}+\Delta P_{4}+\Delta P_{5}$ and $\Delta P_{6}$ for the 4-inch and 6-inch units respectively.

\section{RESULTS}

Fractal measures are estimated using differential pressure signals from different regions in the columns. Procedures to evaluate these metrics are provided in the Appendix. Tolerance parameters used for chaos analysis and RQA are summarized in Table III. Accuracy values are based on manufacturer-provided instrumentation accuracy of $0.14 \%$, while the neighborhood parameter, $\epsilon \sim 5$ times the measurement accuracy of instrumentation was used as recommended by Marwan et al. ${ }^{19}$. In the plots that follow, error bars represent 95\% confidence interval from t-statistic. The readers are urged to exercise caution while making a quantitative comparison of these estimates since hydrodynamic similarity was not preserved across the columns.

\section{Chaos Analysis}

\section{Sample Entropy}

The relationship between average sample entropy $S a m p E n$ and $U / U_{m f}$ is shown in Figure 2. SampEn increases with $U / U_{m f}$ for the most part. Bubbles originating at the distributor propagate upwards as kinematic waves and coalesce with other bubbles. This occurs at a higher frequency as $U / U_{m f}$ increases leading to an increase in SampEn in the dense 
TABLE III. Accuracy and Neighborhood parameters used for chaos analysis and RQA.

\begin{tabular}{c|ccc|ccc}
\hline \hline Region & \multicolumn{3}{|c|}{ Accuracy } & \multicolumn{3}{c}{ Neighborhood } \\
& 2.5 -inch & 4 -inch & 6 -inch & 2.5 -inch & 4 -inch & 6 -inch \\
Distributor & 6.0 & 6.0 & 11.0 & 30.0 & 30.0 & 60.0 \\
Dense region & 10.0 & 4.0 & 4.0 & 50.0 & 20.0 & 20.0 \\
Dilute region & 2.0 & 8.0 & 11.0 & 10.0 & 40.0 & 60.0 \\
Combined & 6.0 & 6.0 & 6.0 & 30.0 & 30.0 & 30.0 \\
\hline \hline
\end{tabular}

region. Larger bubbles after coalescence continue to rise albeit at a lower velocity having a high-pressure nose region and a low-pressure wake. Passage of such bubbles across transducers and their eruption at the interface result in the observed pressure fluctuations in the dilute region. These become more pronounced with increasing $U / U_{m f}$ causing SampEn to increase. Measures from the combined region indicate that the dynamical scales are not linearly super-imposable. SampEn values appear very similar to those in dense region, even though measurements in the combined region include signals from the dilute region.

\section{Hurst Exponent}

Hurst Exponent $(H)$ quantifies system's memory. Data in Figure 3 indicates anti-persistence in differential pressure signals across the distributor region. This is most likely due to combination of piston-like motion of solids near the distributor, self-excited particle interactions and formation of bubbles ${ }^{16}$. All these mechanisms are characterized by short-range temporal features and the corresponding time series have high-frequency components. In the dense region, $H$ continues to indicate anti-persistent behavior in the 4-inch unit while 2.5-inch and 6-inch columns show more persistence in the temporal dynamics. In the dilute region, $H$ indicates uniform persistence in all three columns. This imples larger temporal scales corresponding to the eruption of bubbles at the interface compared to the dynamics in the distributor and dense regions. $H$ values in dense, dilute and combined regions are similar in the 2.5-inch and 6-inch columns, while $H$ values in combined region are similar to dense region in the 4-inch column. It is more likely that pressure signals from bubble formation and coalescence dictate the temporal variation relative to the dynamics at the interface in the 4 -inch unit. Besides, $H$ was found to be insensitive to $U / U_{m f}$ in all the units used in 
the current study.

\section{Correlation Dimension}

Correlation dimension $(D)$ is a measure of fractal nature in a time series. A higher value corresponds to tightly clustered points in the phase-space indicating more randomness. Figure 4 shows the response of correlation dimension to the fluidizing gas velocity $U / U_{m f}$. Similar estimates of $D$ are observed in the 2.5-inch and 4-inch units across all regions, while showing negligible dependence on $U / U_{m f}$. Distributor region has higher $D$ for the 6 -inch unit, while not being sensitive to $U / U_{m f}$. In the other regions, $D$ increases with increase in $U / U_{m f}$, while $D$ values in the combined region are lower than those from individual regions in the 6-inch unit. Results suggest more controlled and self-similar bubbling fluidization in this unit, a phenomenon previously observed in pulsed systems ${ }^{8,12,20}$.

\section{Recurrence Quantification Analysis}

Recurrence is a property used to characterize the behavior of a dynamical system in phase-space $^{19}$. Recurrence of a state in a time series is when a point in the trajectory repeats itself, within a suitably selected margin of error or neighborhood parameter $\epsilon$. A recurrence matrix, $R(i, j)$ can be constructed for the time series $\overrightarrow{\mathbf{x}}$ using the definition given by,

$$
R(i, j)= \begin{cases}1, & \|x(i)-x(j)\| \leq \epsilon \\ 0, & \|x(i)-x(j)\|>\epsilon\end{cases}
$$

Indices i,j denote time instances. $R(i, j)$ is an $N X N$ matrix that represents systems' reconstructed phase-space attractor, where $\mathrm{N}$ is the total number of data points in the time series. Zbilut and Webber ${ }^{21}$, Webber and Zbilut ${ }^{22}$ developed recurrence quantification analysis (RQA) to evaluate the morphology of recurrence plots which might otherwise be challenging to interpret visually depending on the extent of non-linearity. RQA provides a probabilistic tool to quantify the arrangement of data on recurrence plots. Properties such as density of recurrent points, lengths of diagonals, vertical and horizontal lines, are used to derive statistical measures, some of which are presented in the analysis that follows. 
The readers are referred to Marwan et al. ${ }^{19}$ for further details regarding RQA. The RQA parameters are obtained using the Python based library PyRQA ${ }^{23}$ in this study. Recurrence plots from experiments are not shown here for the sake of brevity.

\section{Recurrence Rate}

Recurrence rate $(R R)$ is the probability that the difference between two points in a time series falls within the specified neighborhood parameter, $\epsilon$. The magnitude of $R R$ indicates the degree of invariance in the signal being analyzed. A highly chaotic system with numerous overlapping frequencies from pressure signals would correspond to lower values of $R R$ as observed in the distributor region in Figure 5. Solids undergo significant piston-like motion very close to the distributor. Besides, numerous tiny bubbles are formed in this part of the column. Both these sources are characterized by shorter temporal scales and contribute to small but rapid fluctuations in pressure signals. Hence, $R R$ is lower in this region, where sensitivity to $U / U_{m f}$ is negligible. $R R$ in other sections show dependence on $U / U_{m f}$ and follow varying trends. $R R$ is greater in dense region for the 2.5-inch unit, while it is greater for the 6-inch unit in dilute and combined regions. The dense bed is dominated by a continuous cycle of bubble coalescence and breakup, whereas the dilute region is influenced by bubbles erupting at the interface. The corresponding pressure signals have dominant low-frequency components relative to the signals from distributor region. However, the high-frequency content in these signals increase with $U / U_{m f}$ thereby lowering the $R R$ estimates.

\section{Determinism}

Determinism (DET) measures the probability of recurrent points lying along diagonals of a recurrence plot. Diagonals represent periodicity while broken or intermittent period lines represent unstable orbits of attractors in a chaotic system Marwan et al. ${ }^{19}$. DET measures in the distributor region decreases with increasing $U / U_{m f}$ in all the columns used in the current study. This is indicative of higher levels of chaos in this region at higher fluidization velocities. Also, $D E T$ values for the 6 -inch unit are higher across all regions suggesting a more controlled fluidization process compared to the 2.5-inch and 4-inch units. The observations are consistent with chaos measures in Section III 1. 


\section{Trapping Time}

Results presented so far suggest decreased levels of chaos in the 6-inch unit relative to the 2.5-inch and 4-inch units. This is more apparent from Trapping Time $(T T)$, which represents the length of average vertical line in recurrence plot. TT provides an estimate for the average time a system remains "trapped" in a given state or condition. As a consequence, $T T$ measures are lower for a chaotic system. TT values are similar for all three columns in the distributor region as noticed in Figure 7. TT is high in the dense region in the 2.5-inch unit, and in the dilute region in the 6 -inch unit. $T T$ values from differential pressure signals in the combined region are significantly higher for the 6-inch column at lower $U / U_{m f}$, and drop with increasing $U / U_{m f}$ possibly due to vigorous bubbling as well as bubble eruption at the interface between dense bed and freeboard.

\section{CONCLUSIONS}

The work presented highlights the potential of non-linear analysis to characterize different mechanisms in bubbling fluidized beds. Deterministic chaos was confirmed using differential pressure signals from three fluidized bed columns. Fluctuations in these signals arise from formation of bubbles, their passage across pressure transducers and their eruption at the interface. Chaos and recurrence analyses are used to characterize multi-phase flow dynamics in dense and dilute portions of the test section. The distributor region is highly chaotic compared to the rest. Sample Entropy was found to increase with $U / U_{m f}$ for the most part. Hurst Exponent values were insensitive to $U / U_{m f}$, and indicate anti-persistent behavior near the distributor, becoming more persistent along each column. Pressure signals due to bubble formation and coalescence appear to dictate the temporal scales in the combined region relative to interface dynamics in the 4 -inch column. Correlation dimension measures as well as estimates from RQA suggest possible suppression of chaos in the 6-inch unit. Caution must be exercised while drawing further conclusions from the current study since the systems were not hydrodynamically scaled. Finally, estimates in the combined region reveal the extent of non-linearity in these systems based on the fact that the values are not a trivial combination of measures from dense and dilute regions. 


\section{ACKNOWLEDGEMENT}

This work was performed in support of the US Department of Energy's Fossil Energy Crosscutting Technology Research. The work was executed through the NETL Research and Innovation Center's Advanced Reactor Systems Program. Research performed by Leidos Research Support Team staff was conducted under the RSS contract 89243318CFE000003.

\section{DISCLAIMER}

This work was funded by the Department of Energy, National Energy Technology Laboratory, an agency of the United States Government, through a support contract with Leidos Research Support Team (LRST). Neither the United States Government nor any agency thereof, nor any of their employees, nor LRST, nor any of their employees, makes any warranty, expressed or implied, or assumes any legal liability or responsibility for the accuracy, completeness, or usefulness of any information, apparatus, product, or process disclosed, or represents that its use would not infringe privately owned rights. Reference herein to any specific commercial product, process, or service by trade name, trademark, manufacturer, or otherwise, does not necessarily constitute or imply its endorsement, recommendation, or favoring by the United States Government or any agency thereof. The views and opinions

of authors expressed herein do not necessarily state or reflect those of the United States Government or any agency thereof.

\section{CREDIT LINE}

The article has been submitted to Chaos journal. If published, the final version of this would be found at the following link: https://aip.scitation.org/journal/cha.

\section{Appendix: Formulas to estimate chaos and recurrence analysis parameters}

Parameters used in chaos and recurrence analysis are evaluated as follows:

\section{Sample entropy:}

Sample entropy proposed by Richman and Moorman ${ }^{24}$ is used to quantify the complexity 
or information content of a system. It is defined as:

$$
\operatorname{SampEn}(m, r, N)=-\ln \frac{C^{m+1}(r)}{C^{m}(r)}
$$

where, $C$ is the correlation sum, $m$ is the embedded dimensional space (here $m=2$ ) of the set of vectors derived from the system data used to obtain $C$, and $r$ is coordinate difference criteria for comparing the embedded space vectors. For this study, the sample entropy was calculated via the Nolds python library using $r$ values equal to the accuracy values provided in Table III. A more detailed explanation of sample entropy can be found in Zurek et al. ${ }^{25}$.

\section{Hurst Exponent:}

Hurst Exponent is a measure of system's memory, ranging from 0 to 1 ; where values less than 0.5 denote anti-persistent behavior, values greater than 0.5 denote persistent behavior, and 0.5 denotes Brownian motion. Persistent behavior here refers to whether local increasing/decreasing value trends in system data will continue in the short term. Hurst Exponent is obtained from the rescaled-ranged function following the work of Cabrejos and Klinzing ${ }^{26}$, given by:

$$
R / S=(\alpha \tau)^{H}
$$

where $R$ is the range (max value - min value) of the signal, $\mathrm{S}$ is the standard deviation, $\alpha$ is a proportionality constant, $\tau$ is time period, and $\mathrm{H}$ is the Hurst Exponent. $\mathrm{H}$ is obtained form the slope of log-log plot between $R / S$ and $\tau$. For this study, Hurst Exponent was calculated via the python library $\operatorname{Nolds}^{27}$.

\section{Correlation Dimension:}

Correlation dimension is a measure of fractal dimension of a system often used to characterize strange attractors. Correlation dimensions in this study were calculated using Nolds ${ }^{27}$, in which the underlying algorithm is based on the approach of Grassberger and Procaccia ${ }^{28}$. In summary, the correlation sum $C^{m}(r)$ is calculated as a function of separation distance $r$, where an embedded dimension value of $m=2$ is used. The correlation dimension is then

obtained from the plot between $\ln \left(C^{m}(r)\right)$ and $\ln (r)$ using linear fit. 


\section{Recurrence rate:}

The recurrence matrix is constructed using 1 . The recurrence rate (RR) is defined as the percentage of recurrent points that fall within specified neighborhood. It is obtained using the following expression:

$$
R R=\frac{1}{N^{2}} \sum_{i, j=1}^{N} R(i, j)
$$

A value of 0 indicates no matching points while a value of 1 means all points in the time se-

ries fall within the specified neighborhood. Values used for different sections in the columns are summarized in Table III.

\section{Determinism:}

Determinism (DET) is calculated as the proportion of recurrent points that form diagonal lines given by,

$$
D E T=\frac{\sum_{l=l_{\min }, N}^{N} l P(l)}{\sum_{l=1, N}^{N} l P(l)}
$$

$l$ is the length of diagonal, $l_{\min }$ is the minimum length of line (usually 2 ) and $P(l)$ is the probability of line having length $l . D E T$ is typically high for periodic systems and reduces as they become more chaotic.

\section{Trapping time:}

Trapping time (TT) measures the time period which indicates how long a system remains trapped in a specific state. It is calculated as,

$$
T T=\frac{\sum_{v=v_{\min , N}}^{N} v P(v)}{\sum_{v=v_{\min }, N}^{N} P(v)}
$$

The system is expected to be more stationary when the value of TT is larger.

\section{REFERENCES}

${ }^{1}$ L. R. Glicksman, "Scaling relationships for fluidized beds," Chemical Engineering Science 39, 1373 - 1379 (1984). 
${ }^{2}$ F. Franca, M. Acikgoz, R. Lahey, and A. Clausse, "The use of fractal techniques for flow regime identification," International Journal of Multiphase Flow 17, 545 - 552 (1991).

${ }^{3}$ T. Al-Wahaibi and P. Angeli, "Transition between stratified and non-stratified horizontal oil-water flows. part i: Stability analysis," Chemical Engineering Science 62, 2915 - 2928 (2007).

${ }^{4}$ M. Duponcheel, S. Mimouni, S. Fleau, and Y. Bartosiewicz, "Experimental and numerical investigations of a two-phase wavy flow," Nuclear Engineering and Design 321, 199-218 (2017).

${ }^{5}$ A. Vaidheeswaran and M. Lopez de Bertodano, "Stability and convergence of computational eulerian two-fluid model for a bubble plume," Chemical Engineering Science 160, $210-226(2017)$.

${ }^{6}$ A. Vaidheeswaran, A. Clausse, W. D. Fullmer, R. Marino, and M. Lopez de Bertodano, "Chaos in wavy-stratified fluid-fluid flow," Chaos: An Interdisciplinary Journal of Nonlinear Science 29, 033121 (2019).

${ }^{7}$ R. J. Wilson, P. A. Delamere, F. Bagenal, and A. Masters, "Kelvin-helmholtz instability at saturn's magnetopause: Cassini ion data analysis," Journal of Geophysical Research: Space Physics 117 (2012), 10.1029/2011JA016723.

${ }^{8}$ D. V. Pence and D. E. Beasley, "Chaos suppression in gas-solid fluidization," Chaos: An Interdisciplinary Journal of Nonlinear Science 8, 514-519 (1998).

${ }^{9}$ G.-B. Zhao and Y.-R. Yang, "Multiscale resolution of fluidizedbed pressure fluctuations," AIChE Journal 49, 869-882 (2003), https://aiche.onlinelibrary.wiley.com/doi/pdf/10.1002/aic.690490407.

${ }^{10}$ P. Blomgren, A. Palacios, B. Zhu, S. Daw, C. Finney, J. Halow, and S. Pannala, "Bifurcation analysis of bubble dynamics in fluidized beds," Chaos: An Interdisciplinary Journal of Nonlinear Science 17, 013120 (2007), https://doi.org/10.1063/1.2712309.

${ }^{11}$ W. D. Fullmer and C. M. Hrenya, "The clustering instability in rapid granular and gas-solid flows," Annual Review of Fluid Mechanics 49, 485-510 (2017).

${ }^{12}$ J. E. Higham, M. Shahnam, and A. Vaidheeswaran, "On the dynamics of a quasi-twodimensional pulsed-fludized bed," (2018), arXiv:1809.05033 [physics.flu-dyn].

${ }^{13}$ A. Gel, A. Vaidheeswaran, J. Musser, and C. H. Tong, "Toward the Development of a Verification, Validation, and Uncertainty Quantification Framework for Granular and Multiphase Flows - Part 1: Screening Study and Sensitivity Analysis ," Journal of Verification, 
Validation and Uncertainty Quantification 3 (2018).

${ }^{14}$ B. Gopalan, M. Shahnam, R. Panday, J. Tucker, F. Shaffer, L. Shadle, J. Mei, W. Rogers, C. Guenther, and M. Syamlal, "Measurements of pressure drop and particle velocity in a pseudo 2-d rectangular bed with geldart group d particles," Powder Technology 291, 299 -310 (2016).

${ }^{15}$ A. Vaidheeswaran, F. Shaffer, and B. Gopalan, "Statistics of velocity fluctuations of geldart a particles in a circulating fluidized bed riser," Phys. Rev. Fluids 2, 112301 (2017).

${ }^{16} \mathrm{X} . \mathrm{Bi}$, Flow regime transitions in gas-solid fluidization and transport, Ph.D. thesis (1994).

${ }^{17}$ D. Geldart, "Types of gas fluidization," Powder Technology 7, 285 - 292 (1973).

${ }^{18}$ A. Rao, J. S. Curtis, B. C. Hancock, and C. Wassgren, "The effect of column diameter and bed height on minimum fluidization velocity," AIChE Journal 56, 2304-2311 (2010), https://aiche.onlinelibrary.wiley.com/doi/pdf/10.1002/aic.12161.

${ }^{19}$ N. Marwan, M. C. Romano, M. Thiel, and J. Kurths, "Recurrence plots for the analysis of complex systems," Physics Reports 438, 237 - 329 (2007).

${ }^{20}$ A. Vaidheeswaran, J. E. Higham, and M. Shahnam, "Analysis of a small-scale pulsedfluidized bed," in 71st Annual Meeting of the APS Division of Fluid Dynamics (American Physical Society, 2018).

${ }^{21}$ J. P. Zbilut and C. L. Webber, "Embeddings and delays as derived from quantification of recurrence plots," Physics Letters A 171, 199 - 203 (1992).

${ }^{22}$ C. L. Webber and J. P. Zbilut, "Dynamical assessment of physiological systems and states using recurrence plot strategies," Journal of Applied Physiology 76, 965-973 (1994), pMID: 8175612, https://doi.org/10.1152/jappl.1994.76.2.965.

${ }^{23}$ T. Rawald, M. Sips, and N. Marwan, "Pyrqa - conducting recurrence quantification analysis on very long time series efficiently," Computers and Geosciences 104, 101 - 108 (2017).

${ }^{24}$ J. S. Richman and J. R. Moorman, "Physiological time-series analysis using approximate entropy and sample entropy," American Journal of PhysiologyHeart and Circulatory Physiology 278, H2039-H2049 (2000), pMID: 10843903, https://doi.org/10.1152/ajpheart.2000.278.6.H2039.

${ }^{25}$ S. Zurek, P. Guzik, S. Pawlak, M. Kosmider, and J. Piskorski, "On the relation between correlation dimension, approximate entropy and sample entropy parameters, and a fast algorithm for their calculation," Physica A: Statistical Mechanics and its Applications 391, $6601-6610(2012)$. 
${ }^{26}$ F. J. Cabrejos and G. E. Klinzing, "Characterization of dilute gas-solids flows using the rescaled range analysis," Powder Technology 84, 139 - 156 (1995).

${ }^{27}$ C. Schölzel, "Nonlinear measures for dynamical systems," (2019).

${ }^{28}$ P. Grassberger and I. Procaccia, "Characterization of strange attractors," Phys. Rev. Lett. 50, 346-349 (1983). 
(a)

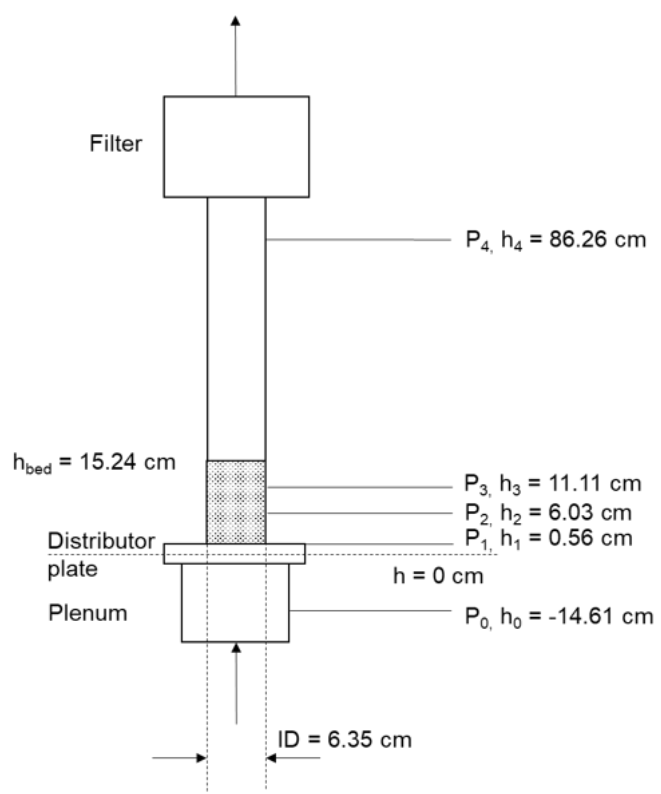

(b)

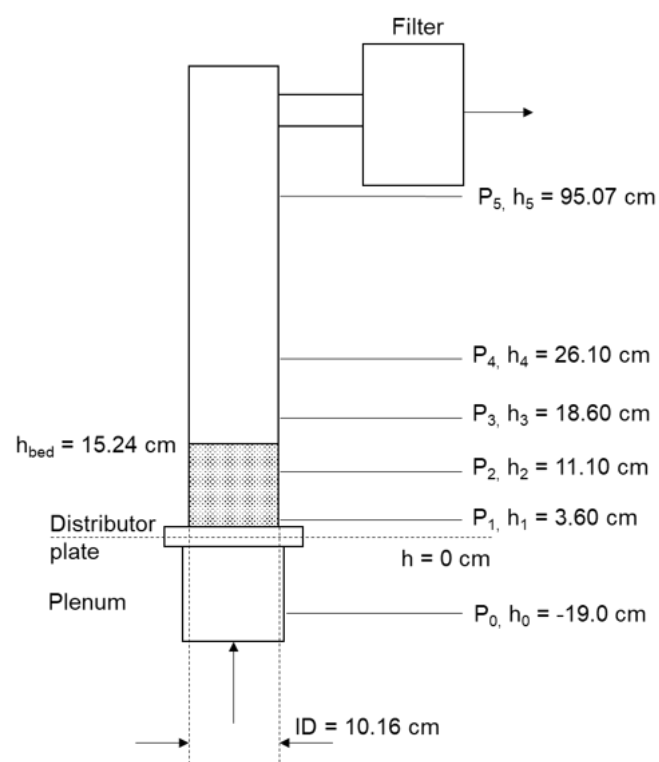

(c)

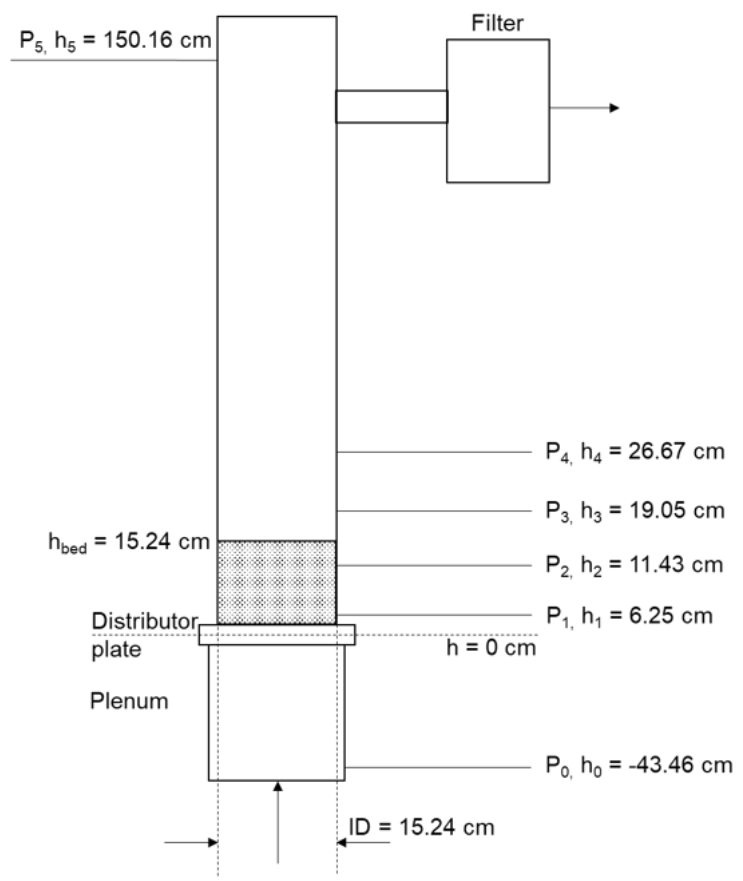

FIG. 1. Schematic of cylindrical columns used in this study having internal diameters of 2.5-inch (a), 4-inch (b) and 6-inch (c). 

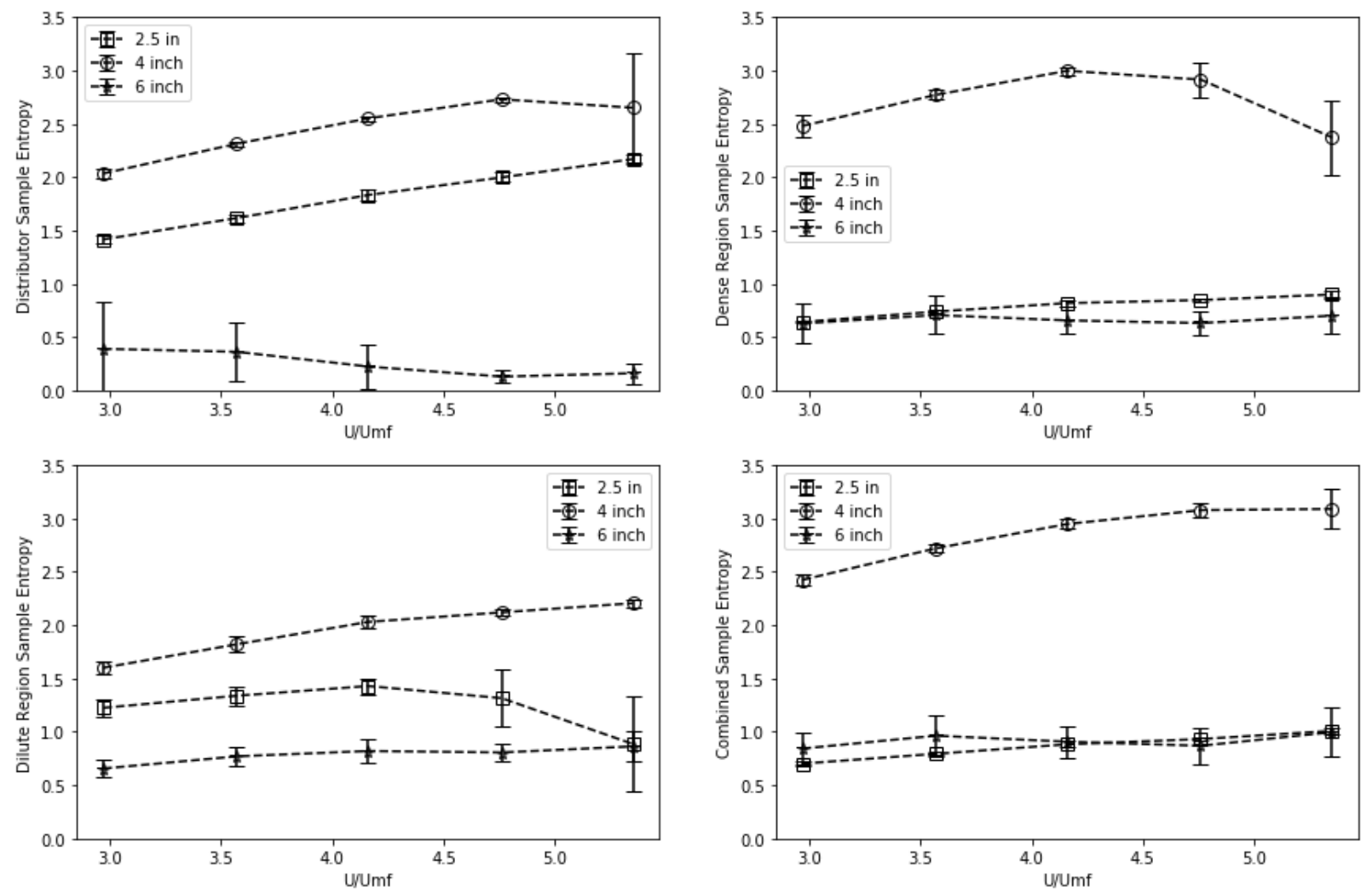

FIG. 2. Sample entropy as a function of $U / U_{m f}$ 

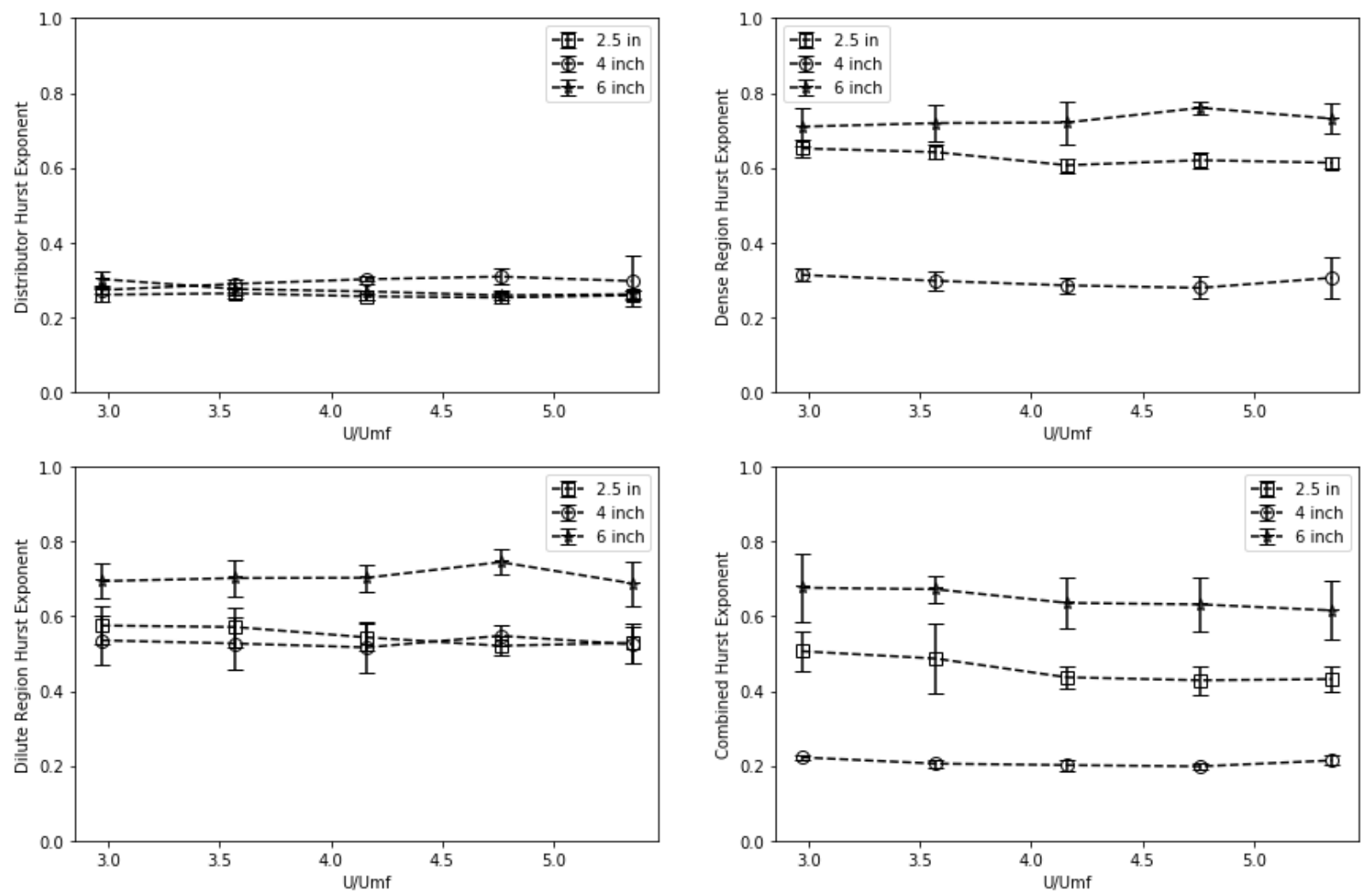

FIG. 3. Hurst Exponent as a function of $U / U_{m f}$ 

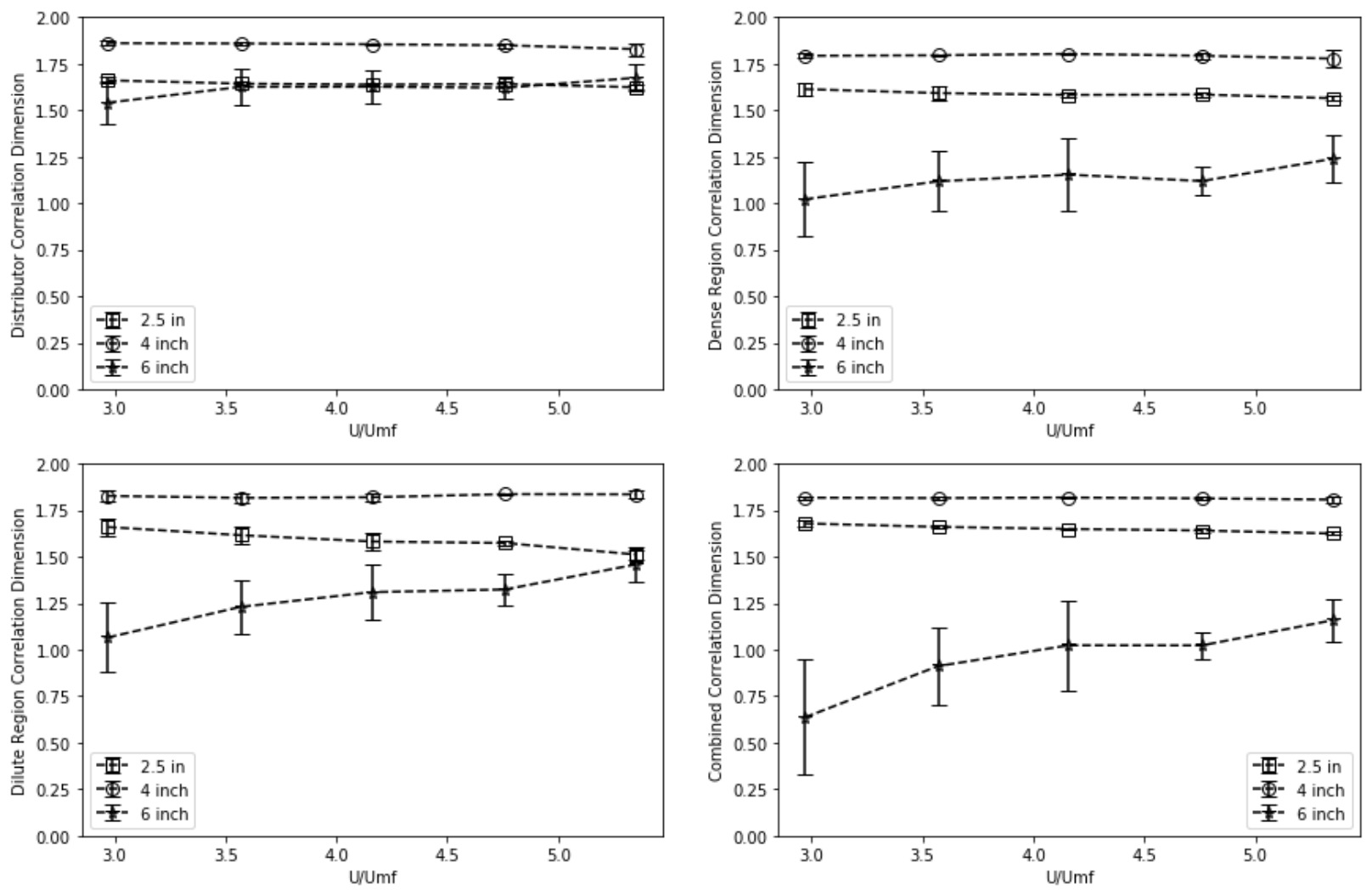

FIG. 4. Correlation dimension as a function of $U / U_{m f}$ 

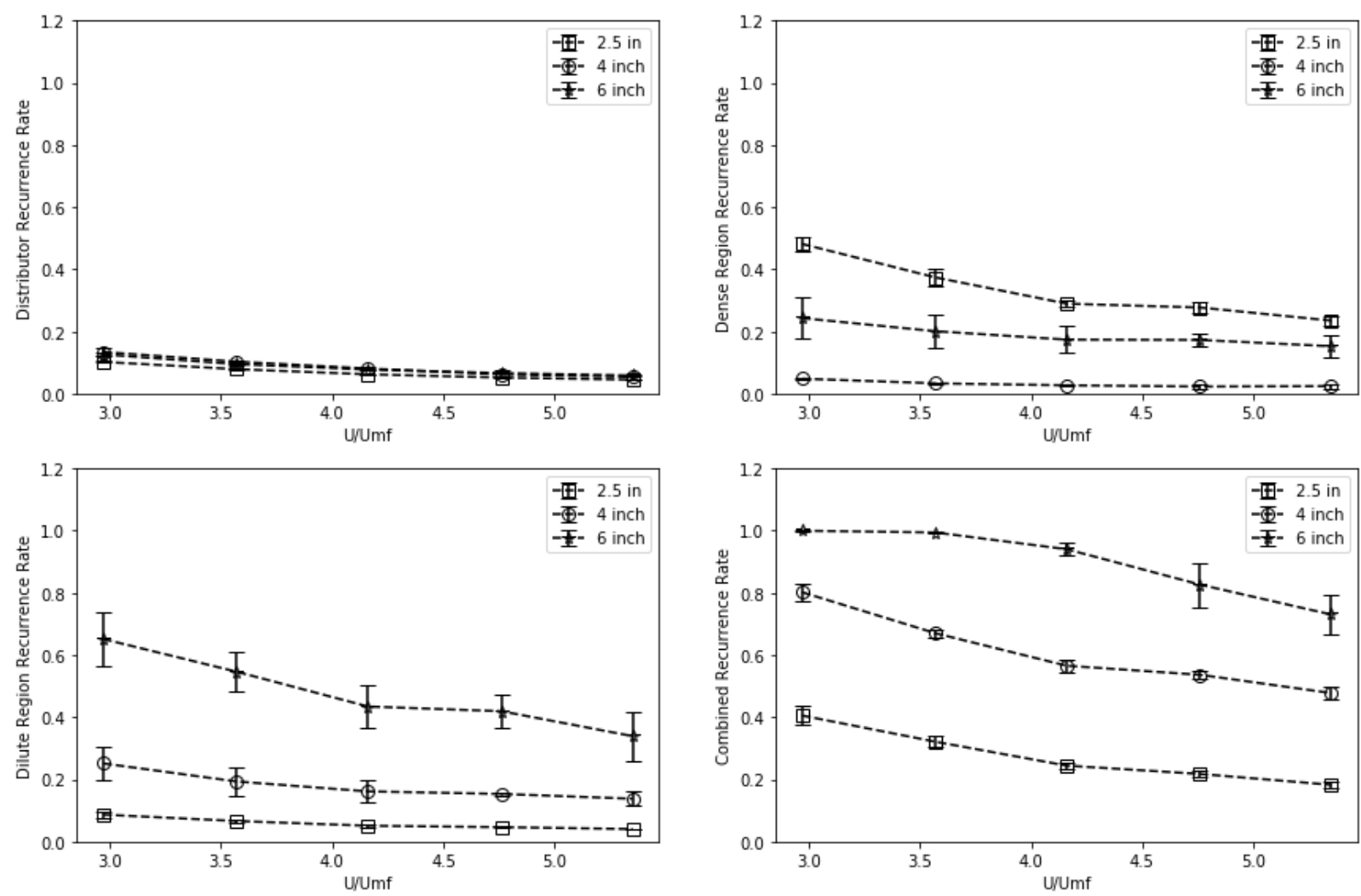

FIG. 5. Recurrence rate as a function of $U / U_{m f}$ 

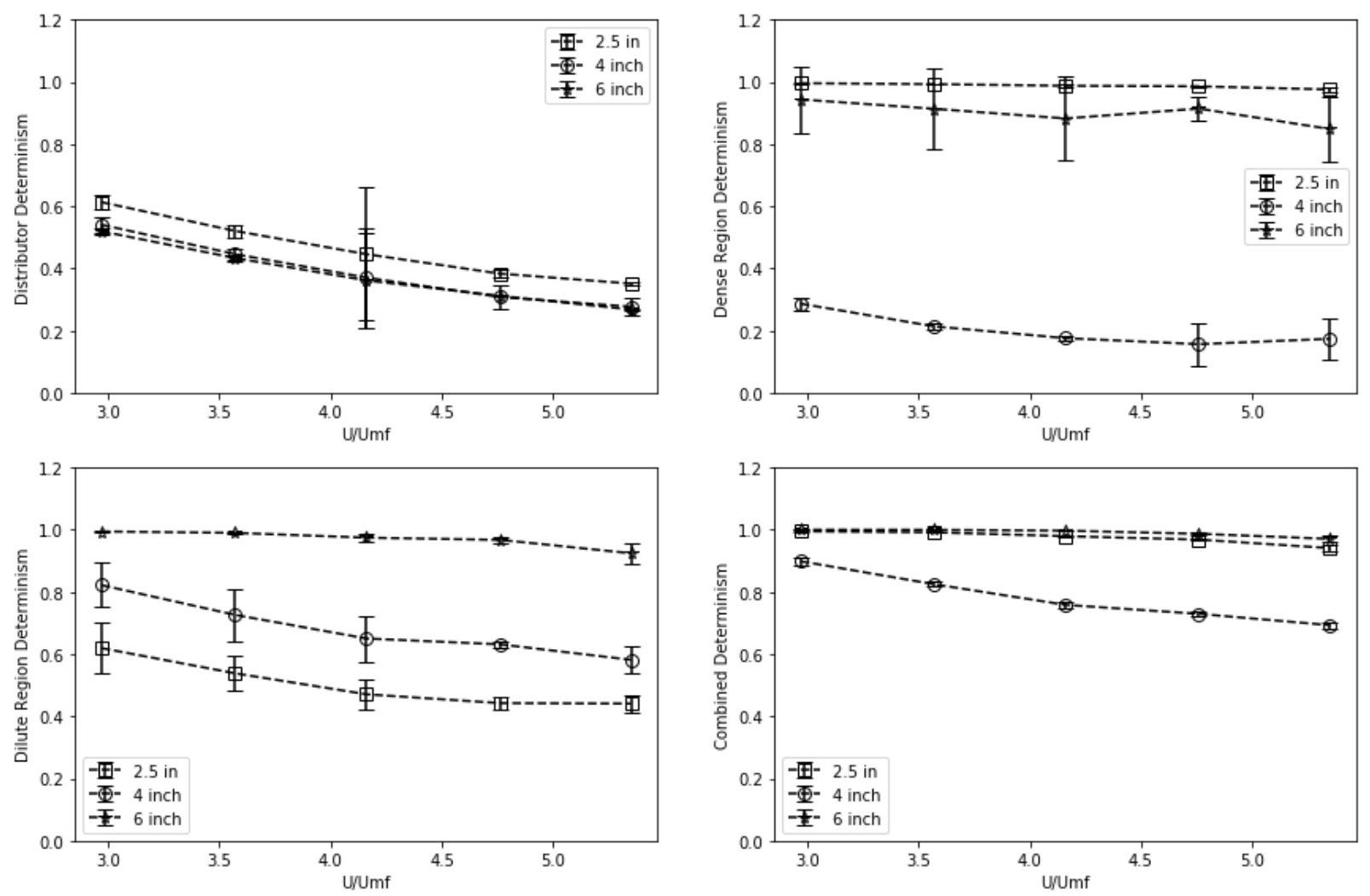

FIG. 6. Determinism as a function of $U / U_{m f}$ 

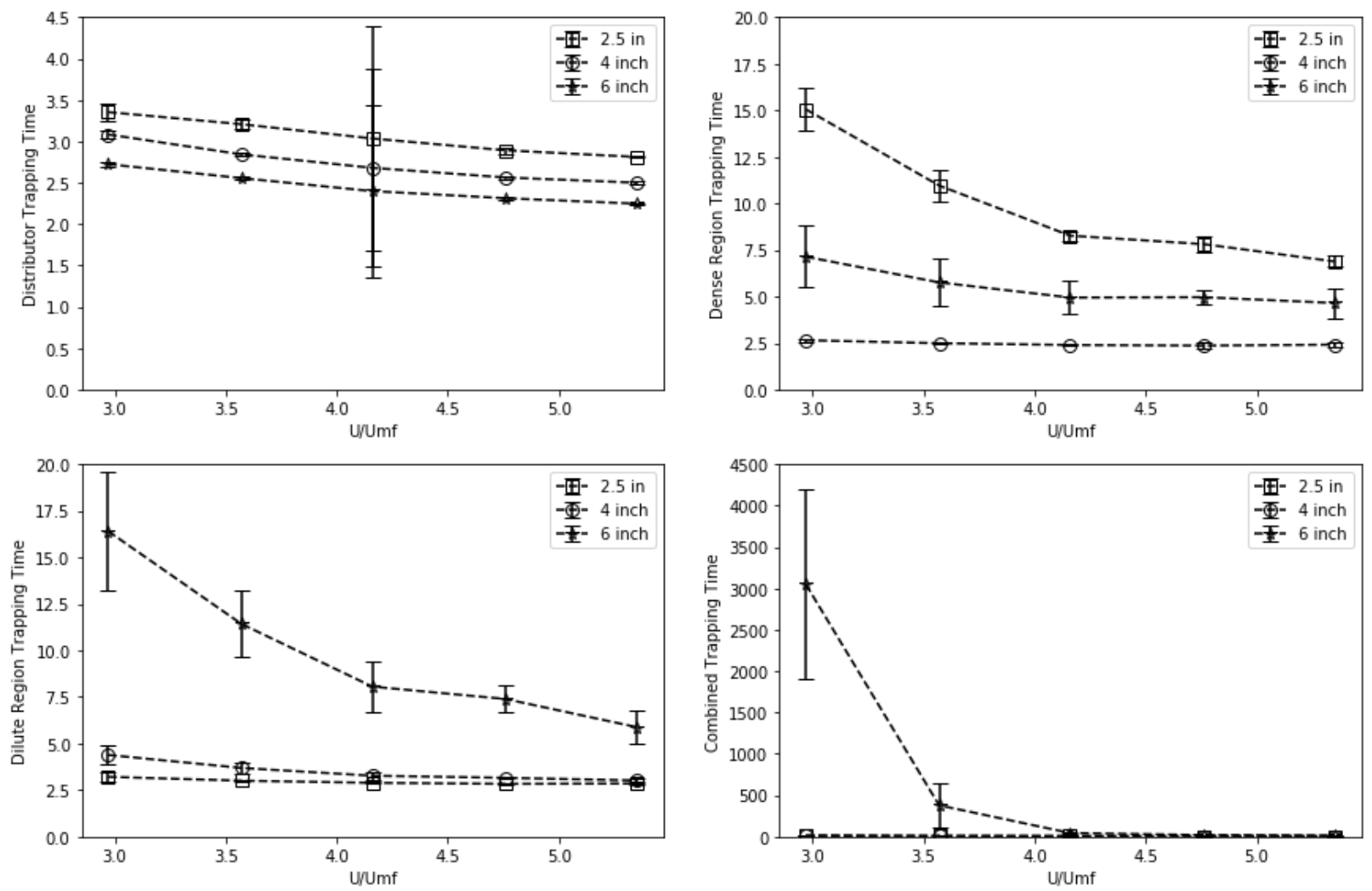

FIG. 7. Trapping time as a function of $U / U_{m f}$ 